in weed species, nor is it attributable to lack of effort in attempting to find suitable biological control agents. For example, the creeping thistle (Cirsium arvense) is a troublesome weed of pastures in Britain and many other countries, both in Europe and North America. Following the work of Zwölfer on the insect predators of this weed, a chrysomelid beetle, Haltica carduorum, which is a native of southern Europe, was introduced into two British localities in 1969 (Claridge et al., Entomologist, 103, $210 ; 1970$ ).

The outcome of these introductions is now described by C. R. B. Baker, R. L. Blackman and M. F. Claridge (J. Appl. Ecol., 9, 819; 1972). Adult beetles imported from France were originally released in June 1969 at Silwood Park, Berkshire, and Llantwit Major, Glamorgan. They bred during the summer, but few survived the 1969 1970 winter. Those which did survive were first seen in mid-April, when the thistles had just begun regrowth after winter dormancy. Further introductions were made in summer 1970, but once again survival was poor.

Detailed studies of these populations showed that egg production was low and mortality was high at all stages in the life history of the beetle. Laboratory studies were carried out on the environmental requirements of the insect and it was found that there was a strong positive correlation between the rate of egg production by each female and temperature over the range $14^{\circ}$ to $22^{\circ} \mathrm{C}$. Egg mortality in the field was around 30 per cent when day temperatures were more than $20^{\circ} \mathrm{C}$, but rose to 100 per cent if $15^{\circ} \mathrm{C}$ was not exceeded. Mortality among larvae was also high at low temperatures. Winter survival of adults was not studied in the laboratory.

The geographical distribution of Haltica carduorum in southern Europe coincides with situations where day temperatures in excess of $20^{\circ} \mathrm{C}$ can be expected regularly during the summer. Evidently the British climate is not so adverse that the completion of the life cycle is totally inhibited in this species, but it does seem to be detrimental to the maintenance of a viable population. Mean temperatures of $13^{\circ}$ to $17^{\circ} \mathrm{C}$, which were recorded in the field during these trials, are evidently sub-optimal for several stages in the life history of Haltica.

An effective biological control agent is one which is specific in its predation, which significantly reduces its prey population and which can maintain viable populations under the prevailing climatic conditions. Evidently Haltica carduorum fails on the third count; its effectiveness on the second count is also open to question. There are predators of Cirsium arvense with more northerly distributions and which are absent from Britain for reasons other than climatic ones, such as isolation by the post-glacial eustatic rise in sea level which has severed Britain from the Continent. The search for an efficient biological control agent of the creeping thistle must now centre upon these species.

\section{GAS DISCHARGES}

\section{Computing Streamers}

GALLIMBERTI reports in a recent issue of Journal of Physics D (5, 2179; 1972) that he has constructed a quantitatively successful model of the propagation of a streamer discharge in a gas which makes use of a simple energy balance criterion. In the past the predictions of streamer models have not been particularly accurate.

A streamer is a weakly ionized and slightly luminous channel which starts from the positive electrode and has at its tip a high concentration of positive charge. The streamer grows as shown in the diagram-photoelectrons produced in front of the tip by photons each generate their own small ionization avalanches by virtue of the local field around the streamer tip. In air at atmospheric pressure, for example, a field of more than $26 \mathrm{kV} \mathrm{cm}{ }^{-1}$ is necessary for avalanche development to be viable.

Unfortunately the equations governing the development of a series of avalanches are, in practice, impossible to solve numerically; Gallimberti makes the simplifying assumption that the avalanches in front of the streamer tip can be represented by a single avalanche which starts immediately ahead of the streamer, somewhere along the line $\mathrm{A}$ but within the active region. The problem is then to calculate where the equivalent avalanche starts. Gallimberti uses as the basis of this calculation the energy balance equation

$$
W_{\mathrm{g}}+W_{\text {pot }}=W_{1}
$$

where the parameters are respectively the gain of energy as a consequence of the applied field, the difference between the potential energies of the streamer tip and the sphere of positive ions created during the development of the equivalent avalanche, and the total loss of energy during the formation of the new avalanche.

During the computing process the lengthening of the streamer caused by each successive equivalent avalanche was reckoned to be twice the radius of

\title{
Orbiting Systems, Mass Loss, and Gravity Pulses
}

Theoretrical physicists continue to be intrigued by the problem of how large quantities of gravitational radiation might be produced in plausible astronomical situations. In next Monday's Nature Physical Science (February 19), Jackson and Matzner describe the effects of sudden loss of mass from a primary object on a hypothetical small companion in orbit around the primary. Because the mass of the primary $(M)$ changes abruptly, the secondary (mass $m$ ) experiences a changing acceleration. This "jerk", defined as the third time derivative of position, or rate of change of acceleration, can become very large for large $M$. Such a situation provides a powerful, if short lived, mechanism for the production of gravitational radiation, because the third derivative of the quadrupole moment of the pair $m, M$ becomes large, and the rate of production of quadrupole gravitational radiation depends on the square of this derivative.

Some curious features emerge from the details of the analysis which Jackson and Matzner have made. If $\dot{M}$ is sufficiently large, the gravitational radiation shows similarities to radiation from a particle in a circular orbit; but in the case of the jerk mechanism information about the central gravity field-that is, about the nature of the mass $M$-is contained in the field equations. This seems to be a result of the requirement that the speed of light is constant; the information that $M$ is changing must be transmitted throughout $M$, and this will take a different time for diffuse objects, compact stars, or black holes.

Could this jerk mechanism have a bearing on the seemingly large flux of gravitational radiation reported by Weber to be coming from the galactic centre? If the loss of mass occurs at the galactic centre, from a compressed object, and if the Sun is taken as the secondary particle, then the flux rate observed at the Earth should be only $10^{-7}$ times that claimed by Weber. "It is tempting," say Jackson and Matzner, "to attempt to sum the contributions of many particles $m$ surrounding a central body undergoing mass loss." This would only help with the observational problem, however, if the jerk radiation originates at the small particles and in some sense in phase ; then a reverberation could sweep the cloud of small particles as the information about the loss of mass from $M$ spreads outwards.

Because the work described in next Monday's Nature Physical Science is based on the weak field approximation, the intriguing speculations encouraged by this last possibility should really hang fire until similar calculations have been carried out for strong fields. Jackson and Matzner promise that such calculations are now under way. 